\title{
Thermal Desorption of Hydrogen From Graphane
}

\author{
L. A. Openov* and A. I. Podlivaev \\ Moscow Engineering Physics Institute (State University), 115409 Moscow, Russia \\ * E-mail: LAOpenov@mephi.ru
}

\begin{abstract}
The process of hydrogen desorption from graphane (graphene sheet saturated by hydrogen adsorbed from both sides) has been studied using the method of molecular dynamics. The temperature dependences of the time of desorption onset for various hydrogen coverages on graphene are calculated and the corresponding activation energies in the Arrhenius equation are determined. It is established that graphane exhibits a rather high thermal stability that makes possible its use in two-dimensional electronics even at room temperature. For the same reason, graphane can hardly be considered as a promising hydrogen storage material for fuel cells.
\end{abstract}


Graphene, representing a monolayer sheet of carbon atoms, draws the attention of both basic science (as a medium featuring massless Dirac fermions) and practical applications (as a material for nanoelectronics) [1]. It is also of interest to study the properties of graphene derivatives (e.g., in the form of nanoribbons [2]), including those obtained by its chemical modification with polymers, nitrogen, hydrogen, etc. Sofo et al. [3] theoretically predicted the existence of graphane, representing a graphene sheet saturated by hydrogen adsorbed from both sides, and quite recently this prediction was experimentally confirmed $[4]$.

Graphane (unlike graphene) is a dielectric and, in principle, it can also be used in nanoelectronics. The relatively large content (7.7 mass \%) of hydrogen in graphane suggests that it probably can be used in hydrogen power engineering. In assessing the possibilities of the practical usage of graphane, it is necessary first to study its thermal stability with respect to hydrogen desorption and thus determine the range of permissible temperatures for potential applications. This Letter presents the results of an investigation of the desorption of hydrogen from graphane, which was numerically simulated using the method of molecular dynamics.

The initial sample of graphene was modeled by a hexagonally packed monolayer fragment consisting of 54 carbon atoms. In order to reduce the influence of the finite size of the sample (edge effects), the dangling bonds of $s p$-hybridized edge carbon atoms were saturated by hydrogen. This fragment was converted into graphane by attaching one hydrogen atoms to each carbon atom alternatively from the two sides of the monolayer. As a result, we obtained a $\mathrm{C}_{54} \mathrm{H}_{72}$ model cluster of graphane schematically depicted in Fig. 1. In this cluster, all atoms occur in the $s p^{3}$-hybridized state [4] (in contrast to 
the $s p^{2}$-hybridization in graphene) and are shifted in the transverse direction (off-plane) by $0.2-0.3 \AA$ depending on the position relative to the center of the cluster. The $\mathrm{C}-\mathrm{H}$ and $\mathrm{H}-\mathrm{H}$ bond lengths in the graphane fragment are 1.10 and $1.50-1.53 \AA$, respectively. Note that it is necessary to distinguish between 18 hydrogen atoms used to passivate the sample edge and 54 hydrogen atoms forming the graphane structure: the former $\mathrm{H}$ atoms are present in both graphene and graphane models, whereas the latter are present only in graphane; in macroscopic graphane samples of increasing size, the relative fraction of the former $\mathrm{H}$ atoms tends to zero, while that of the latter tends to unity.

The aim of this study was to determine the temperature dependence of the time necessary for the detachment of one $\mathrm{H}$ atom (of the total of 54 hydrogen atoms entering into the graphane structure) from the $\mathrm{C}_{54} \mathrm{H}_{72}$ cluster. This corresponds to the removal of a $1 / 54 \approx 2 \%$ fraction of $\mathrm{H}$ atoms from a macroscopic sample of graphane, that is, to the formation of a relatively large number of defects (sufficient to significantly change the electrical characteristics). This task was solved using the method of molecular dynamics $[5,6]$. At the initial moment, all atoms were randomly imparted velocities and displacements so that the total momentum and moment of the entire cluster were zero. Then, the forces acting on atoms were calculated and the classical Newton equations of motion were numerically integrated using the velocity Verlet method with a temporal step of $t_{0}=2.72 \times 10^{-16} \mathrm{~s}$. The total system energy (a sum of the potential and kinetic energies) remained unchanged, which corresponded to a microcanonical ensemble (a system not in thermal equilibrium with the environment) $[5,6]$. The relative motion of atoms was characterized by the dynamic temperature $T$ that was determined from the following relation: $\left\langle E_{k i n}\right\rangle=\frac{1}{2} k_{B} T(3 n-6)$ where $\left\langle E_{k i n}\right\rangle$ is the kinetic energy averaged over the time, $k_{B}$ is 
the Boltzmann constant, and $n$ is the number of atoms in the system. This approach was successfully used previously for the qualitative explanation of experimental data on the fragmentation of $\mathrm{C}_{60}$ fullerene [6].

An important question is how to calculate the forces acting on atoms at each step of molecular dynamics. In fact, this procedure reduces to calculating the total system energy as a function of the coordinates of component atoms. Solving this task by the ab initio methods requires considerable computational facilities and allows the evolution of a system involving $\sim 100$ atoms to be traced for a rather short time on the order of $\sim 10 \mathrm{ps}$ which is insufficient to accumulate a necessary statistics. For this reason, we employed a nonorthogonal tight-binding model [7], which reasonably compromise between the strict ab initio methods and oversimplified approaches based on classical interatomic interaction potentials. The proposed model rather well describes both small carbon clusters (e.g. fullerenes [7]) and hydrocarbon structures (e.g., cubane $\mathrm{C}_{8} \mathrm{H}_{8}$ [8]) and macroscopic systems [7]. We believe that the method of molecular dynamics in combination with a tight-binding potential provides a quite adequate description of the thermal stability of graphane, similar to the results obtained by applying the same approach to cubane [8].

Evolution of the $\mathrm{C}_{54} \mathrm{H}_{72}$ cluster was traced to the moment of detachment of the first $\mathrm{H}$ atom at various temperatures in the range of $T=1300-3000 \mathrm{~K}$. The results presented in Fig. 2 show that as the temperature decreases, the desorption onset time $\tau$ exponentially increases from $\sim 0.1$ ps to $\sim 10$ ns. The plot of $\ln (\tau)$ versus $1 / T$ is well approximated by a straight line, which indicates that the process of hydrogen desorption can be described in terms of the following standard Arrhenius relationship:

$$
\tau^{-1}(T)=A \cdot \exp \left[-\frac{E_{a}}{k_{B} T}\right]
$$


where $A$ is the temperature-independent (or weakly dependent) frequency factor and $E_{a}$ is the activation energy (which can be determined from the slope of the straight line in Fig. 2). Statistical analysis of the results of modeling yields $E_{a}=(2.46 \pm 0.17) \mathrm{eV}$ and $A=(2.1 \pm 0.5) \times 10^{17} \mathrm{~s}^{-1}$. By substituting these $E_{a}$ and $A$ values into formula (1), we can evaluate $\tau$ at low temperatures, which are inaccessible for computer simulations because of extremely long necessary computation times. At $T=300 \mathrm{~K}$, this estimation yields a macroscopic value of $\tau \sim 10^{24} \mathrm{~s}$, which is evidence for the possibility of using graphane in nanoelectronic devices operating at room temperature. As the temperature increases to $600 \mathrm{~K}$, the value of $\tau$ drops to $\sim 1000 \mathrm{~s}$. These results do not contradict the recent data [4], according to which the complete desorption of hydrogen from graphane was achieved by annealing it for one day in argon at $T=700 \mathrm{~K}$. It should be noted that, in view of the exponential character of relation (1), any refinement of the $E_{a}$ value can lead to rather significant changes in the values of $\tau$. Therefore, the above values of $\tau$ at different temperatures should be considered as rough estimates.

A detailed analysis of evolution of the model $\mathrm{C}_{54} \mathrm{H}_{72}$ cluster showed that the desorption of hydrogen from various parts of the cluster takes place at approximately equal probability . This fact agrees with the results of calculations of the heights $U$ of energy barriers for the desorption of $\mathrm{H}$ atoms. These values were determined by studying the potential energy hypersurface of the given cluster as a function of the coordinates of component atoms (see [9]). The barrier height $U$ amounts to $2.4 \mathrm{eV}$ at the center of the cluster and increases to $2.7 \mathrm{eV}$ at the periphery. As expected, these values are close to the desorption activation energies $E_{a}$. It should be noted that, in the course of simulations, we observed two cases of the desorption of $\mathrm{H}_{2}$ molecules rather than atomic hydrogen. 
In order to study the dependence of the rate of desorption on the degree of graphene coverage by hydrogen (graphane corresponds to the total coverage, whereby the number $\mathrm{N}_{H}$ of nonpassivating hydrogen atoms is equal to the number $\mathrm{N}_{C}$ of carbon atoms in the sample), we have also determined $\tau$ as a function of $T$ for two other systems representing graphene with a half coverage $\left(\mathrm{N}_{H}=\mathrm{N}_{C} / 2, \mathrm{C}_{54} \mathrm{H}_{45}\right.$ cluster $)$ and with a single adsorbed $\mathrm{H}$ atom $\left(\mathrm{N}_{H}=1, \mathrm{C}_{54} \mathrm{H}_{19}\right.$ cluster $)$. The corresponding values of the desorption activation energy $\left(E_{a}=(1.74 \pm 0.17)\right.$ and $E_{a}=(1.86 \pm 0.14) \mathrm{eV}$, respectively $)$ proved to be smaller compared to the value obtained for graphane, in agreement with the lower barriers for desorption $(U=(1.2-1.9) \mathrm{eV}$, increasing toward the periphery) and with the general tendency of a decrease in the thermodynamic stability of hydrocarbons with decreasing relative content of hydrogen [3]. These results indicate that the time of the total desorption of hydrogen from graphane is determined by the initial stage, in which the hydrogen content decreases by $(1-10) \%$.

It is interesting to note that, in simulating the $\mathrm{C}_{54} \mathrm{H}_{45}$ and $\mathrm{C}_{54} \mathrm{H}_{19}$ clusters, we have repeatedly observed the fracture of the carbon framework much before the hydrogen desorption event. This behavior agrees with the data of experiments [4], in which an increase in the temperature of graphane annealing above a certain level led to damage of the graphene framework. Taking into account that (i) this effect is not observed in the $\mathrm{C}_{54} \mathrm{H}_{72}$ cluster and (ii) the rate of hydrogen desorption increases with decreasing content of $\mathrm{H}$ atoms, it may be recommended to perform the initial stage of annealing at a higher temperature and then slightly cool the sample.

In conclusion, let us briefly consider the possibility of using graphane in fuel cells for electric car engines. In addition to meeting the requirements of high hydrogen content 
(more than 6 mass \%) and stability at room temperature, it is necessary that the fuelcell material would be capable of rapidly (within $\sim 1 \mathrm{~s}$ ) and almost completely release hydrogen at a temperature not exceeding $400 \mathrm{~K}$ [10]. According to the results of our investigation, graphane does not obey the last requirement. This is probably related to the strong covalent $\mathrm{C}-\mathrm{H}$ bonds which, on the one hand, ensure a high thermal stability of hydrogen chemisorbed on carbon nanostructures [10] and, on the other hand, sharply decrease the rate of hydrogen desorption. Therefore, the most promising directions of the practical use of graphane are apparently related to nanoelectronics. In this context, it will be of interest to study the phenomenon of thermoactivated migration of hydrogen through the graphene/graphane interface. Should this boundary remain atomically sharp at not very low temperatures, it will be possible to attempt at creating various nanoelectronic devices by selectively adsorbing hydrogen on graphene sheets and nanoribbons.

\section{References}

[1] A. K. Geim and K. S. Novoselov, Nature Mater. 6, 183 (2007).

[2] Z. Chen, Y.-M. Lin, M. J. Rooks, and P. Avouris, Physica E 40, 228 (2007).

[3] J. O. Sofo, A. S. Chaudhari, and G. D. Barber, Phys. Rev. B 75, 153401 (2007).

[4] D. C. Elias, R. R. Nair, T. M. G. Mohiuddin et al., Science 323, 610 (2009).

[5] I. V. Davydov, A. I. Podlivaev, and L. A. Openov, Fiz. Tverd. Tela (St. Petersburg) 47, 751 (2005) [Phys. Solid State 47, 778 (2005)].

[6] L. A. Openov and A. I. Podlivaev, Pisma Zh. Eksp. Teor. Fiz. 84, 73 (2006) [JETP Lett. 84, 68 (2006)]. 
[7] M. M. Maslov, A. I. Podlivaev, and L. A. Openov, Phys. Lett. A 373, 1653 (2009).

[8] M. M. Maslov, D. A. Lobanov, A. I. Podlivaev, and L. A. Openov, Fiz. Tverd. Tela (St. Petersburg) 51, 609 (2009) [Phys. Solid State 51, 645 (2009)].

[9] A. I. Podlivaev and L. A. Openov, Pisma Zh. Eksp. Teor. Fiz. 81, 656 (2005) [JETP Lett. 81, 533 (2006)].

[10] J. Li, T. Furuta, H. Goto et al., J. Chem. Phys. 119, 2376 (2003). 


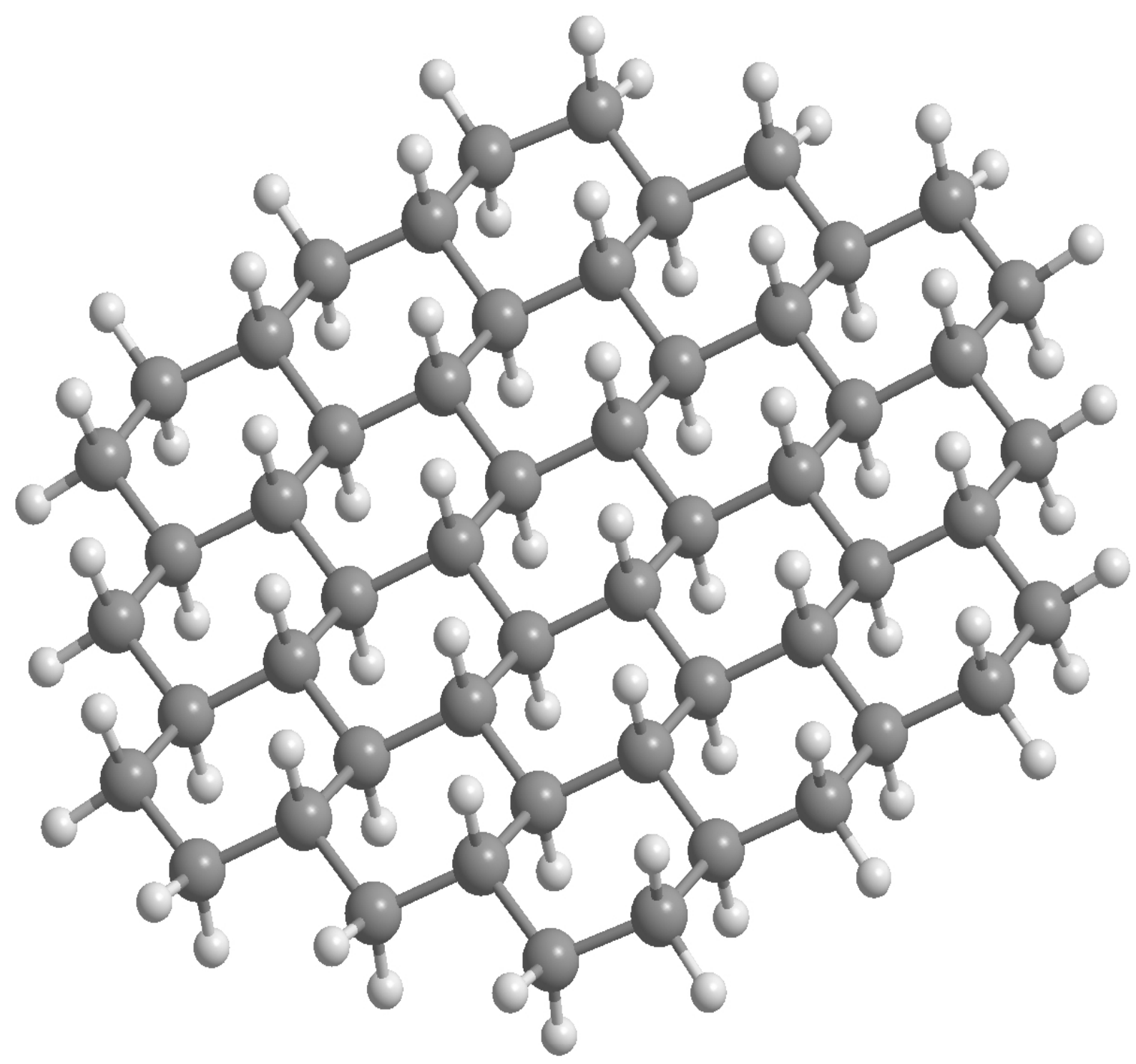

Fig. 1. $\mathrm{C}_{54} \mathrm{H}_{72}$ cluster modeling a graphane fragment. Large and small balls represent carbon and hydrogen atoms, respectively. 


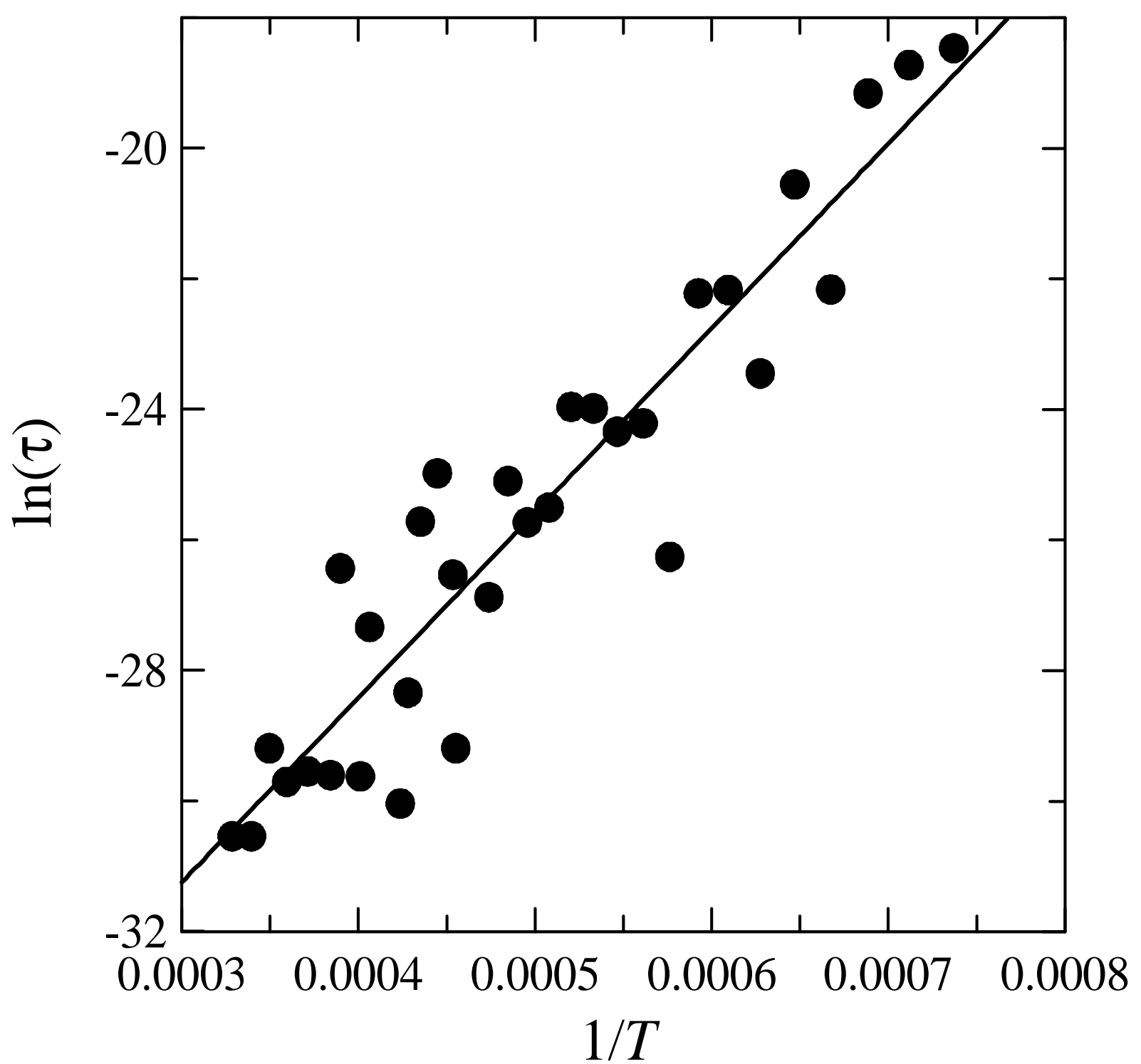

Fig. 2. Plot of the logarithm of desorption time $\tau$ [s] of one $\mathrm{H}$ atom from the $\mathrm{C}_{54} \mathrm{H}_{72}$ cluster versus inverse temperature $T[\mathrm{~K}]$. Points present the results of model calculations, solid line shows the linear approximation by least squares. 